\title{
Evaluación de la predicción de aceleraciones debidas al tránsito peatonal en una pasarela en servicio
}

\author{
Evaluation of acceleration estimates due to pedestrian \\ loading on an in-service footbridge
}

L. de Sebastián ${ }^{(*)}$, I. M. Díaz ${ }^{(* *)}$, C. M. Casado ${ }^{(*)}$, A. V. Poncela(***), A. Lorenzana ${ }^{(* * *)}$

\section{RESUMEN}

En este artículo se evalúan algunos modelos de carga de peatones en la pasarela peatonal del Museo de la Ciencia de Valladolid siguiendo estos pasos: (i) identificación experimental de los parámetros modales usando diversos métodos para la estimación de la masa modal, (ii) estimación de la respuesta de aceleración utilizando algunos modelos de carga dados por normativas, y (iii) desarrollo de pruebas experimentales y comparación de los valores de aceleración medidos con los estimados. Se ha estudiado la influencia de los peatones en las propiedades modales incluyéndose este efecto en la estimación de las aceleraciones y la mejora de las mismas. Así, este trabajo es un ejemplo de análisis de las vibraciones de una pasarela en servicio poniendo especial atención a la bondad de las estimaciones comparadas con los registros reales y a la influencia de los peatones en dichas estimaciones.

\section{2-158}

Palabras clave: Pasarela peatonal; Análisis dinámico; Sistema peatones-estructura; Modelo de carga de peatones.

\section{SUMMARY}

Pedestrian load models on the Valladolid Science Museum Footbridge have been evaluated in this paper following these steps: (i) experimental identification of the modal parameters using several methods for the modal mass estimation, (ii) estimation of acceleration response through pedestrian models given by codes, and (iii) development of experimental tests and comparison of acceleration records with acceleration estimates. The influence of pedestrians on the structural modal properties has been studied and its effects have been considered into the acceleration estimates, leading to better estimates. Then, this paper is an example of vibration analysis of an in-service footbridge focusing on the performance of vibration estimates as compared with experimental measures and on the influence of pedestrians on those estimates.

Keywords: Footbridge; Dynamic analysis; Pedestrian-structure system; Pedestrian load model.

\footnotetext{
(*) CARTIF Centro Tecnológico, Valladolid (España).

(**) Universidad Politécnica de Madrid (España).

(***) Universidad de Valladolid (España).

Persona de contacto/Corresponding author: jesseb@cartif.es (J. de Sebastián)
}

Recibido/Received: 27 mar 2012 Aceptado/Accepted: 22 jun 2012 Publicado online/ Published online: 01 jul 2013 


\section{INTRODUCCIÓN}

El avance en los materiales y en las tecnologías de construcción ha propiciado el desarrollado de estructuras civiles más ligeras y esbeltas, construidas con menos material, menos mano de obra y menos tiempo de construcción (1) (2). Por otro lado, estas estructuras son más susceptibles de vibrar ante cargas producidas por el viento, automóviles o personas. Más concretamente, estructuras tales como forjados de grandes luces, pasarelas peatonales, escaleras esbeltas o graderíos, pueden ser excitadas por los usuarios al realizar actividades como andar, correr o saltar. La excitación producida por los usuarios podría alcanzar niveles de vibración excesivos, sobrepasando los valores de confort recomendados en las normativas. El factor decisivo en el dimensionamiento de este tipo de estructuras suele ser el estado límite de servicio de vibraciones (ELSV).

Existen varias guías de cálculo y normativas en las que se incluyen diversos modelos de cargas producidas por humanos, y entre ellos se encuentran aquéllos que modelan un flujo o tránsito de peatones continuo, que es el estado de carga más representativo. Estos modelos pueden ser empleados en el dimensionamiento de la estructura, pero en general no se confía demasiado en ellos ya que no existen muchos ejemplos de verificación en estructuras ya construidas (3). Por tanto, es interesante comprobar como se comportan estos modelos en estructuras en servicio.

La influencia de los peatones parados o sentados (pasivos) en el comportamiento dinámico de las estructuras se ha estudiado en numerosos trabajos de investigación (4) (5); sin embargo no está tan clara la influencia que tienen los peatones activos (andando, corriendo o saltando) en las propiedades dinámicas del conjunto peatones-estructura. En algunos trabajos previos se ha visto que esta influencia podría ser importante (6) (7) y debería considerarse en cualquier predicción teórica previa. Así, en este trabajo se estudia esa influencia en las propiedades dinámicas con objeto de valorar la importancia que tiene su consideración en la fase de cálculo.

En el presente trabajo se comentan las principales normativas en vigor junto con algunas de las guías de cálculo de pasarelas peatonales publicadas hasta la fecha. Posteriormente, se analiza la dinámica de la pasarela peatonal del Museo de la Ciencia de Valladolid (PMC) obteniéndose sus parámetros modales. A continuación se obtienen las predicciones de la respuesta de aceleración de la misma utilizando tres documentos: el Eurocódigo 5 (8), la ISO 10137 (9) y la guía SETRA (10). Por último, se realizan pruebas experimentales y se comparan los resultados estimados utilizando algunos de los modelos recogidos en dichos documentos con los valores experimentales.

\section{NORMATIVAS Y GUÍAS DE CÁLCULO DE PASARELAS PEATONALES}

Cuando un proyectista de estructuras tiene la tarea de analizar, en la fase de cálculo, el ELSV de una pasarela peatonal, éste dispone de un gran número de documentos en forma de normas, instrucciones, recomendaciones y guías nacionales e internacionales. Este apartado trata de dar una visión conjunta de todos estos documentos.

En el caso de España, en 1996 el Ministerio de Fomento publicó las recomendaciones para el proyecto de puentes metálicos sobre carreteras RPM 95 (11) y su homóloga para puentes mixtos RPX 95 (12). Estas recomendaciones, en la parte del ELSV, se limitan a establecer el valor máximo de aceleración vertical $\left(0,5 \sqrt{ }\left(f_{0}\right)\left(\mathrm{m} / \mathrm{s}^{2}\right)\right.$, siendo $f_{0}$ la frecuencia fundamental del vano) para puentes con tránsito de peatones y pasarelas. Además, en ausencia de cálculos específicos, establecen que la aceleración máxima es admisible si se cumple una condición de flecha máxima estática. En 1998 se publicó la primera edición de la instrucción sobre las acciones a considerar en el proyecto de puentes de carretera IAP 98 (13), incluyendo pasarelas peatonales. Esta instrucción sólo plantea la realización de pruebas de carga dinámicas en aquellas pasarelas en las que las vibraciones puedan afectar a su funcionalidad. En 2011 se revisó este documento denominándose IAP 11 (14), en el que se incluyó un nuevo apartado de "Estado límite de vibraciones en pasarelas peatonales". En este apartado se establecen: (i) unos rangos de frecuencia críticos para los que será necesario realizar estudios dinámicos (vertical/longitudinal: $[1,25-4,60] \mathrm{Hz}$, lateral $[0,50-1,20] \mathrm{Hz}), y$ (ii) unas circunstancias que, con independencia de los valores de las frecuencias naturales, implican la realización de estudios dinámicos (luz > $50 \mathrm{~m}$, anchura útil > $3 \mathrm{~m}$, tipología singular, nuevos materiales, ubicación en zona urbana con previsible tráfico intenso de peatones). En el apartado de pruebas de carga, se establece que cuando la pasarela peatonal esté dentro de los criterios para la realización de estudios dinámicos se incluirá el proyecto de prueba de carga dinámica, para tal efecto se podría consultar el apartado correspondiente del documento "Recomendaciones 
para la realización de pruebas de carga de recepción de puentes de carretera" (15) que publicó el Ministerio de Fomento en 1999.

Por otro lado, la instrucción de hormigón estructural EHE-08 (16) y la de acero estructural EAE (17) en sus últimas versiones también establecen el ELSV en pasarelas peatonales. La EHE-08 fija el mismo valor de aceleración de confort que en la RPX 95 y RPM 95, y establece la necesidad de comprobar el valor de la flecha estática debida a un peatón de $750 \mathrm{~N}$ en el punto de máxima flecha para pasarelas con el primer modo de flexión vertical menor de $5 \mathrm{~Hz}$. También indica la necesidad de realizar pruebas de carga dinámicas cuando se prevea la aparición de vibraciones molestas para los usuarios. Por otro lado, la EAE (artículo 38) (estado límite de vibraciones) establece rangos de frecuencia críticos (vertical: [1,6-2,4] $\mathrm{Hz}$ y $[3,5-4,5] \mathrm{Hz}$ y horizontal: [0,6-1,2] $\mathrm{Hz}$ ), y los niveles de vibración máximos que deben cumplirse (vertical: $0,7 \mathrm{~m} / \mathrm{s}^{2}$ cuando un peatón de $750 \mathrm{~N}$ ande o corra; horizontal: $0,2 \mathrm{~m} / \mathrm{s}^{2}$ en condiciones de uso normal y $0,4 \mathrm{~m} / \mathrm{s}^{2}$ en condiciones de aglomeraciones excepcionales). También establece la realización de estudios con modelos de carga correspondientes a peatones individuales y grupos (andando, saltando, etc.) pero no define qué modelos teóricos de carga deben emplearse. Además, estos escenarios de carga deberán acordarse entre el autor del proyecto y el propietario de la pasarela.

Una mención aparte merecen los Eurocódigos. Los eurocódigos no son de obligado cumplimiento, a menos que exista una cláusula de excepción general prevista en el Código Técnico de la Edificación (18). De todos los eurocódigos, los que tratan temas relacionados con la evaluación del ELSV y que pueden ser aplicados a pasarelas peatonales son: la UNE-EN 1990:2003 (19) o Eurocódigo 0 de Bases de cálculo, la UNE-EN 1991-2:2004 (20) o Eurocódigo 1 en su parte de cargas de tráfico en puentes y la UNE-EN 1995-2:2010 (8) o Eurocódigo 5 de estructuras de madera en el apartado de puentes.

Como resumen, la normativa española en relación con las herramientas para la verificación del ELSV en pasarelas peatonales dispone de varios documentos en los que no se definen modelos para las cargas ni se detalla una metodología que ayude en el proceso de cálculo, debiendo ser dichas cargas definidas entre el proyectista de la estructura y el propietario de ésta, por lo que en general es necesario acudir a alguna de las guías de cálculo internacionales existentes.

Aparte de los eurocódigos, existen una serie de normativas internacionales que en muchos casos han sido la base de las normativas nacionales. Ejemplos de éstas son la ISO 2631-1 Mechanical vibration and shock - Evaluation of human exposure to wholebody vibration - Part 1: General requirements (21) de 1985, revisada en 1997 y aceptada por AENOR en 2008 (UNE-ISO 2631). Es también reseñable la ISO 10137 Bases for design of structures: Serviceability of buildings and walkways against vibrations (9) de 1992 y revisada en 2007.

Finalmente, en los últimos años se han publicado guías de pasarelas peatonales con el objeto de facilitar a los proyectistas el proceso de cálculo. Las guías más extendidas son "Guidelines for the design of footbridges" (22) publicada por FIB en 2005, "Assessment of vibrational behaviour of footbridges under pedestrian loading" (10) publicada por Sétra en 2006 y "Design of footbridges" (23) publicada como resultado del proyecto Hivoss en 2008. En estas guías se dan pautas de cómo diseñar y evaluar la respuesta dinámica de las pasarelas en la fase de cálculo. En este sentido, dan formulaciones sencillas de modelos de carga para un peatón, grupos de peatones y flujo continuo de peatones en los que se tiene en cuenta factores de sincronización en función del rango de frecuencias de paso, entre otros aspectos.

En la Figura 1 se muestra una línea temporal con las fechas de publicación de los principales documentos de consulta tanto nacionales (parte superior de la línea) como internacionales (parte inferior de la línea).

\section{IDENTIFICACIÓN DE LOS PARÁMETROS MODALES DE LA ESTRUCTURA}

En esta sección se describe la identificación de los parámetros modales de la PMC. Se realizó un análisis modal operacional (AMO) y un análisis modal experimental
1. Fechas de publicación de documentos nacionales e internacionales relacionadas con el estado de servicio en pasarelas peatonales.

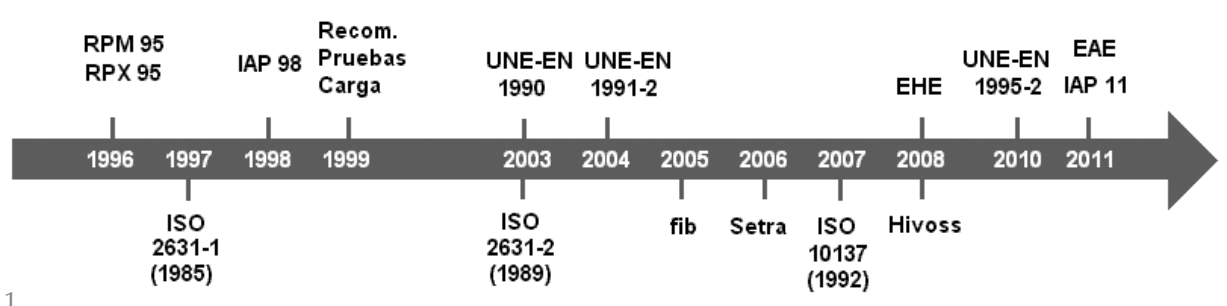



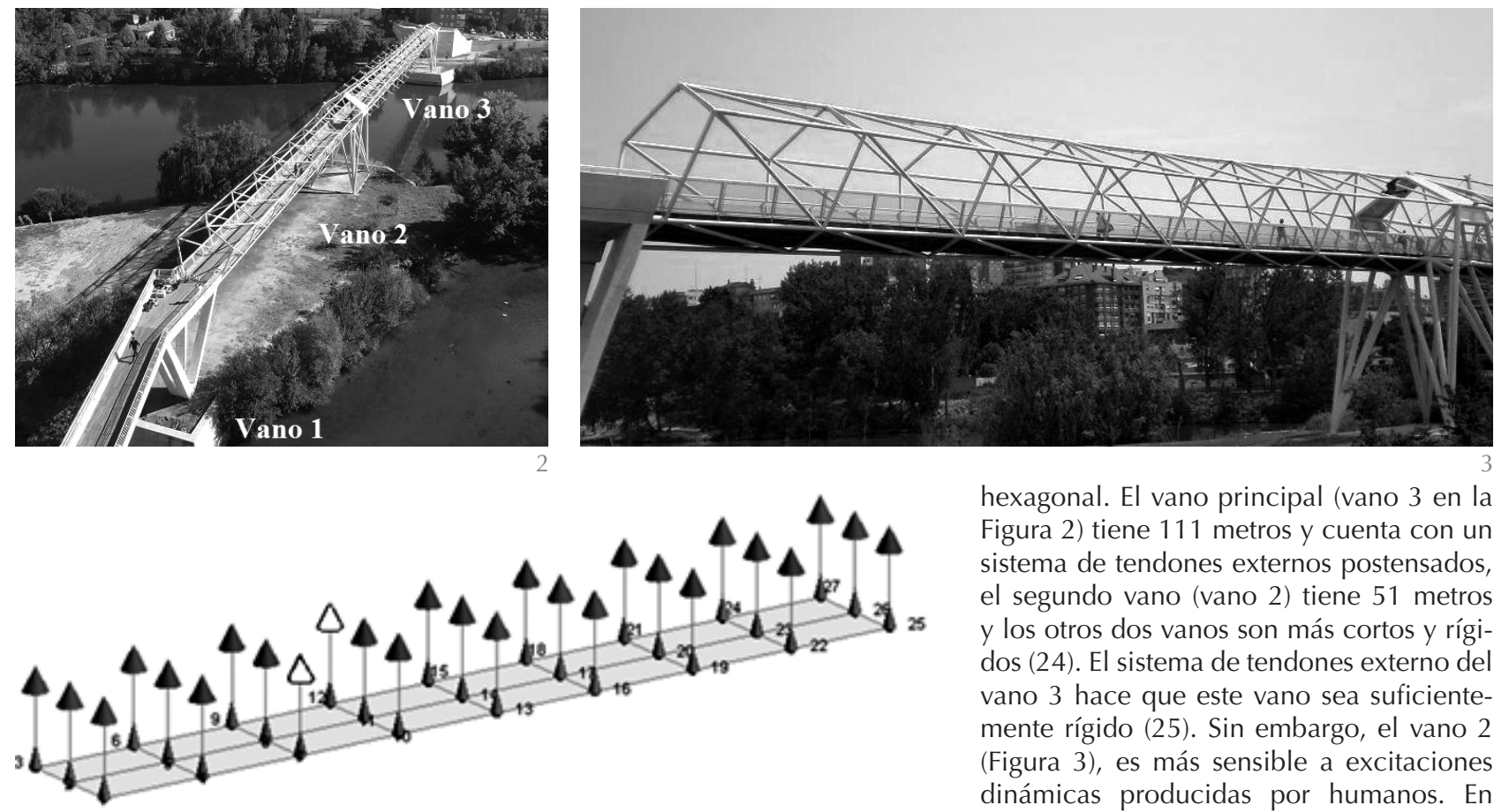

4

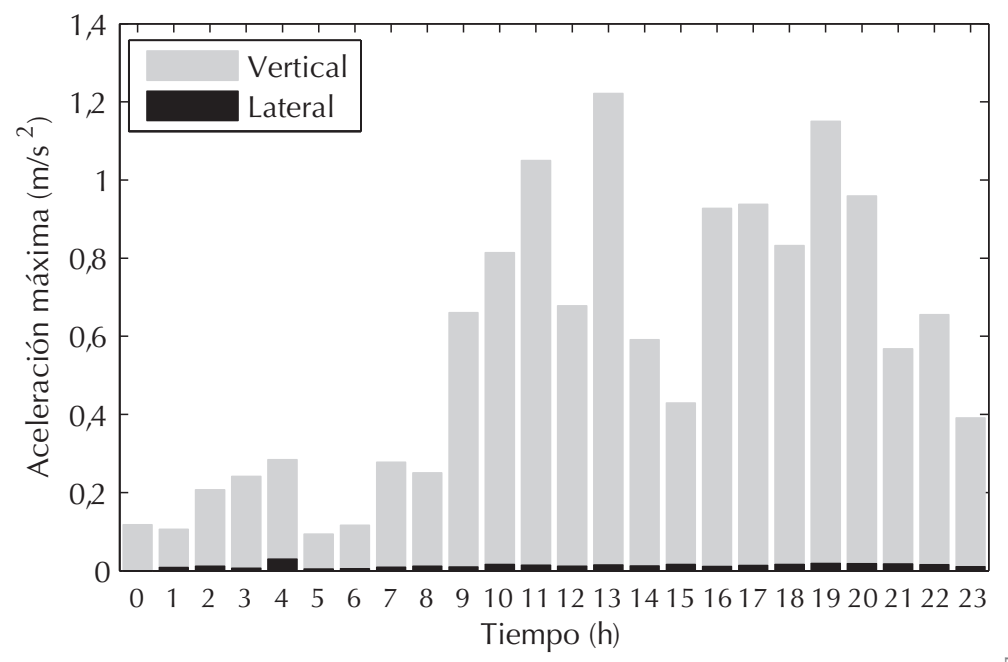

2. Vista general de la estructura en estudio.

3. Vista del vano 2 .

4. Posición de los acelerómetros de medida, con los de referencia en blanco.

5. Ejemplo de valores máximos de aceleración en cada hora durante un día completo.

hexagonal. El vano principal (vano 3 en la
(AME) del vano donde se registran los mayores niveles de vibraciones. Los ensayos se realizaron con un número de peatones variable para estudiar su influencia en los parámetros modales. También se estudió la linealidad de la estructura para descartar que la variación en los parámetros modales fuera debido a un posible comportamiento no lineal.

\subsection{Descripción de la estructura}

La estructura estudiada, situada en Valladolid, es una pasarela peatonal sobre el río Pisuerga que une el Museo de la Ciencia con el centro de la ciudad. Este puente, de 234 metros y construido en el año 2004, está compuesto por cuatro vanos: tres formados por barras metálicas tubulares y uno de hormigón, con tablero de madera. Los tres vanos metálicos tienen sección Figura 2) tiene 111 metros y cuenta con un sistema de tendones externos postensados, el segundo vano (vano 2) tiene 51 metros y los otros dos vanos son más cortos y rígidos (24). El sistema de tendones externo del vano 3 hace que este vano sea suficientemente rígido (25). Sin embargo, el vano 2 (Figura 3), es más sensible a excitaciones dinámicas producidas por humanos. En dicho vano, con una masa estimada de $27.500 \mathrm{~kg}$, se alcanzan niveles de vibración que, según la opinión de los usuarios, a veces son molestos, particularmente cuando un corredor cruza el puente. Así, se decidió estudiar las propiedades dinámicas de este vano.

\subsection{Análisis modal operacional}

Se realizó un AMO del vano 2 para obtener las frecuencias naturales, coeficientes de amortiguamiento y modos de vibración de las primeras frecuencias. Para la realización del AMO se utilizaron cinco acelerómetros móviles y dos de referencia (MMFKS48C-1000 mV/g). Se consideró una malla de tres líneas longitudinales con un total de 27 puntos de medida. Se registraron cinco conjuntos de medidas con un tiempo de adquisición de 12 minutos y una frecuencia de muestreo de $100 \mathrm{~Hz}$. En la Figura 4 se muestra la disposición de los acelerómetros y la posición de los de referencia. Se realizaron análisis espectrales preliminares y registros temporales que indicaron que la vibración vertical era considerablemente mayor que la horizontal, por lo que sólo se midió la respuesta vertical. Como ejemplo, se muestran en la Figura 5 los valores máximos de aceleración medidos (vertical y lateral) en cada hora durante un día completo. En ese día la vibración máxima medida fue de $1,20 \mathrm{~m} / \mathrm{s}^{2}$ en vertical y de $0,03 \mathrm{~m} / \mathrm{s}^{2}$ en lateral. La estimación de los parámetros modales se hizo utilizando el método en el dominio de la frecuencia EFDD (Enhanced Frequency Domain Decomposition). Se estiman los modos a partir de la descomposición en valores singulares de las matrices de densidad espectral de cada una de las 
mediciones y se identifica cada valor singular como un sistema de un grado de libertad. En la Figura 6 se muestran los cuatro primeros valores singulares promediados obtenidos empleando el programa comercial ARTeMIS (26). En la Tabla 1 se muestran los parámetros modales estimados (frecuencias y amortiguamientos modales) y en la Figura 7 los correspondientes modos de vibración. El primer modo, con una frecuencia de $3,51 \mathrm{~Hz}$, podría ser excitado por una persona andando (2. ${ }^{\circ}$ armónico) o corriendo (1. ${ }^{\text {er }}$ o $2 .^{\circ}$ armónico).

Para conocer la variabilidad de sus parámetros modales en servicio se realizó un $\mathrm{AMO}$ de la pasarela con diferentes niveles de ocupación. El análisis modal se llevó a cabo utilizando los acelerómetros de la línea central. El tiempo de adquisición con peatones se incrementó a 30 minutos para eliminar la influencia de éstos en la excitación. Los análisis de realizaron con 1, 2, 3, 4 y 15 peatones caminando a su ritmo, eligiendo tanto la velocidad como la forma de andar y pudiendo adelantar para no influirse unos a otros. Los modos resultantes eran similares a los obtenidos en el $\mathrm{AMO}$ sin peatones, pero se observó un aumento considerable del amortiguamiento. Este comportamiento también se ha documentado en otros trabajos (5) (27) (28) (29). Los resultados estimados se muestran en la Tabla 2. Se aprecia que con 4 peatones casi se dobla el amortiguamiento y con 15 se quintuplica. Este es un parámetro significativo ya que se emplea en la predicción de la respuesta de la estructura (8) (9) (10). Por otro lado, se observó que la frecuencia estimada apenas cambiaba: sólo se reducía muy ligeramente, probablemente debido al efecto de la masa de los peatones (menos del $4 \%$ de la masa del vano).

\subsection{Estimación de la masa modal}

La identificación de los parámetros modales de la estructura utilizando el AMO tiene el inconveniente de no identificar la masa modal, parámetro necesario para realizar las estimaciones de la respuesta. Para la determinación de la masa modal del primer modo se han utilizado tres métodos: el primero, basado en el AME, en la que la fuerza de excitación es conocida; el segundo,

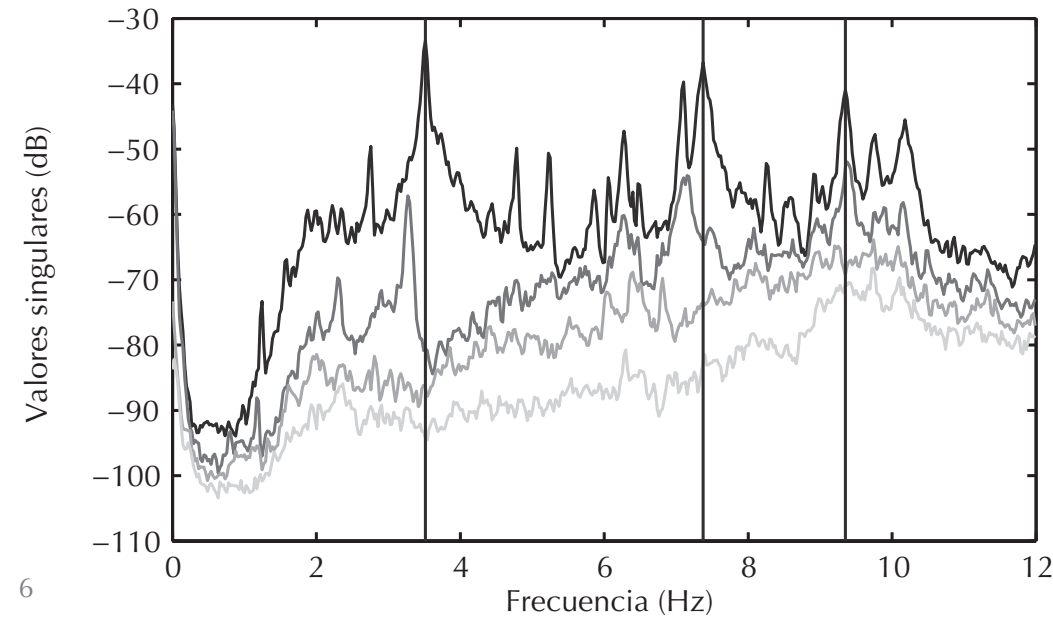

basado en el ajuste del modelo modal utilizando un oscilador (30) y el tercero, basado en el ajuste del modelo modal al aumentar la respuesta de la estructura ante una excitación armónica (31). Los dos últimos no requieren la medición de la excitación.

Para el AME, la fuerza de excitación se generó con un excitador electrodinámico de APS Dynamics modelo 400 en modo inercial colocado en el centro del vano (32). La fuerza inducida por el excitador se calculó midiendo la aceleración de la masa inercial y multiplicándola por su magnitud $(30,4 \mathrm{~kg})$. Se utilizó un barrido continuo en frecuencia entre 2,5 y $4,5 \mathrm{~Hz}$ para excitar el primer modo. Se utilizaron 7 acelerómetros (como los usados en el AMO) colocados en la línea central de la pasarela. En la Figura 8 se muestra la función de respuesta en frecuencia (FRF) entre la respuesta de aceleración de la estructura y la excitación. La masa modal estimada fue de $18.000 \mathrm{~kg}$. Este método tiene la ventaja de conocer la excitación y el inconveniente de que se necesita un excitador capaz de hacer vibrar la estructura.

En el caso de la estimación usando un oscilador se utilizó una masa de 200 kg sintonizada a la primera frecuencia natural $(3,5 \mathrm{~Hz})$. Utilizando los algoritmos en el tiempo y en la frecuencia descritos en (30) se obtuvo una masa modal de aproximadamente $18.000 \mathrm{~kg}$. El inconveniente de este método es que hay que utilizar un oscilador sintonizado a la frecuencia del modo que se quiere estimar lo suficientemente grande
6. Valores singulares promediados de las matrices de densidad espectral de las aceleraciones medidas e identificación de los modos dominantes.

7. Modos de vibración identificados.

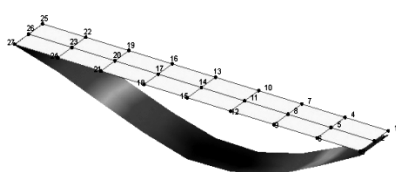

1. ${ }^{\mathrm{er}}$ modo: $3,5 \mathrm{~Hz}$

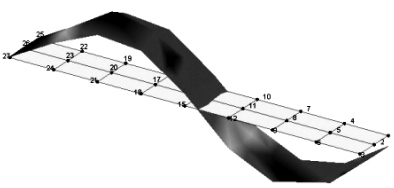

2. ${ }^{\circ}$ modo: $7,4 \mathrm{~Hz}$

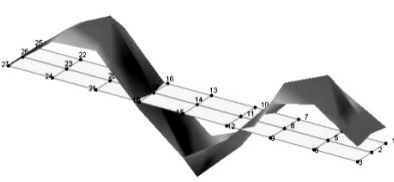

3. ${ }^{\text {er }}$ modo: $9,4 \mathrm{~Hz}$

Tabla 1. Frecuencias naturales y amortiguamientos identificados con el AMO

\begin{tabular}{|c|c|c|c|c|}
\hline Algoritmo & Parámetro & Modo 1 & Modo 2 & Modo 3 \\
\hline \multirow{2}{*}{ EFDD } & Frecuencia $(\mathrm{Hz})$ & 3,51 & 7,41 & 9,46 \\
\cline { 2 - 5 } & Amortiguamiento $(\%)$ & 0,41 & 0,35 & 0,36 \\
\hline
\end{tabular}

Tabla 2. Evaluación de la frecuencia natural y del amortiguamiento

\begin{tabular}{|c|c|c|c|c|c|c|}
\hline Parámetro & Sin peatones & 1 peatón & 2 peatones & 3 peatones & 4 peatones & 15 peatones \\
\hline Frec. $(\mathrm{Hz})^{(1)}$ & 3,519 & 3,519 & 3,516 & 3,516 & 3,515 & 3,514 \\
\hline Amort. $(\%)$ & 0,41 & 0,47 & 0,64 & 0,70 & 0,72 & 2,33 \\
\hline
\end{tabular}




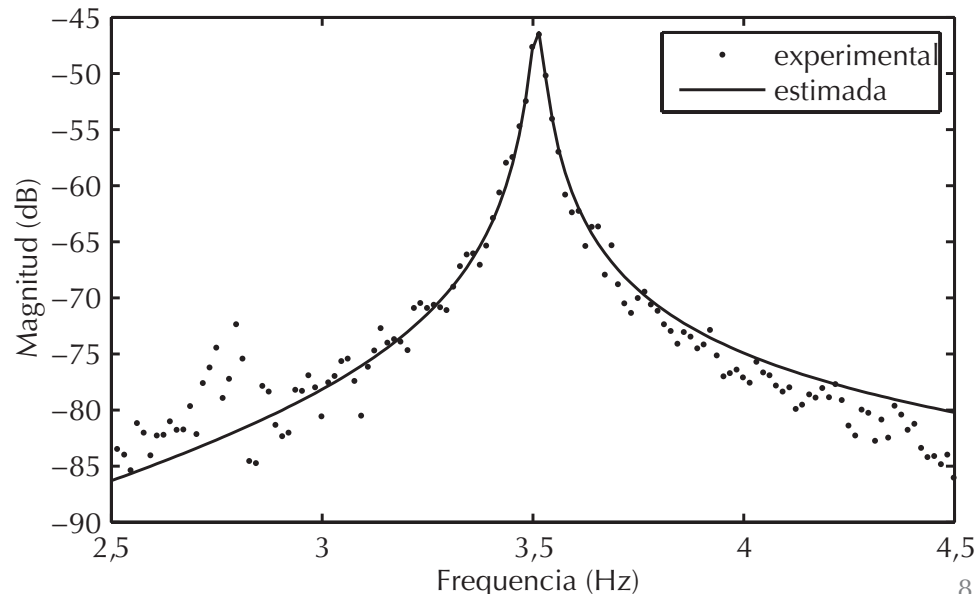

8. Magnitud de la Función de Respuesta en Frecuencia (FRF), en $\mathrm{dB}$ (normalizada a $1 \mathrm{~ms}-2 \mathrm{~N}-1$ ) medida experimentalmente $\mathrm{y}$ estimada.

9. Frecuencia y amortiguamiento del primer modo en función de la amplitud de la respuesta.

para modificar el comportamiento de la estructura. Sin embargo, tiene la ventaja de que no se necesita un actuador.

Para el método basado en el incremento en la respuesta se excitó la pasarela mediante una persona que saltaba, sin levantar los de la masa modal se estimó en 17.500 kg. La ventaja de este método es que sólo se necesita una persona que previamente haya registrado su fuerza en el laboratorio y el inconveniente es que puede que la excitación medida en el laboratorio y la generada en la estructura no sea la misma.

Además de los métodos empleados aquí existen otros métodos relacionados con el AME para calcular la masa modal como por ejemplo el método de adición de masa para calcular el factor de escala (33).

\subsection{Evaluación de la linealidad de la pasarela} pies, a la frecuencia de $3,5 \mathrm{~Hz}$. La fuerza generada por la persona fue medida previamente en el laboratorio usando una placa de fuerza (Kistler 9286AA). Se ajustó el modelo para que la respuesta del sistema ante dicha fuerza fuera igual que la respuesta de la estructura medida. El valor
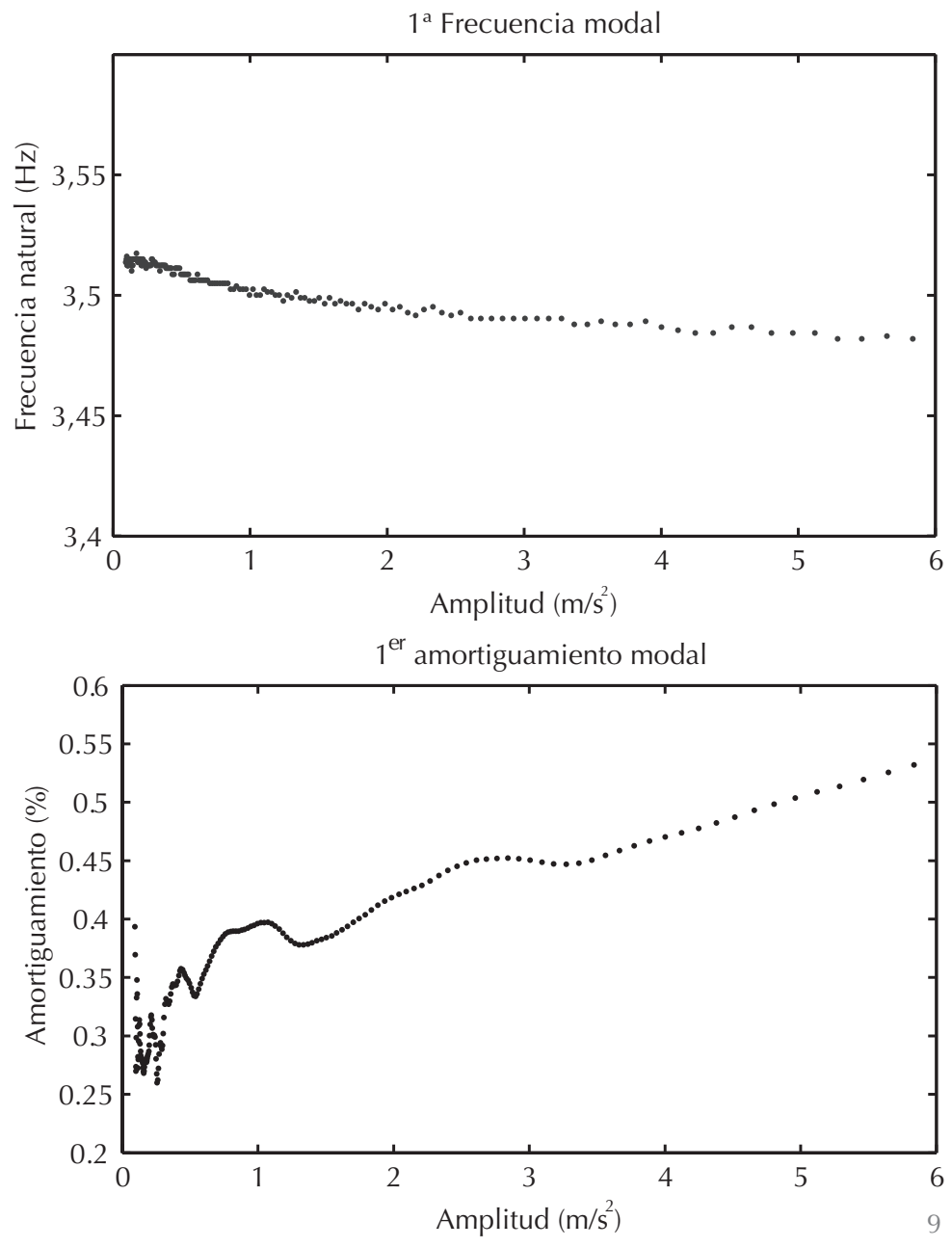

Es conveniente realizar un estudio del comportamiento dinámico bajo diferentes niveles de aceleración para determinar si la estructura se comporta linealmente. La pasarela se excitó mediante un peatón corriendo a la frecuencia de resonancia y se empezó a medir cuando el peatón había abandonado la pasarela para no influir en los parámetros de la estructura. La frecuencia natural y el coeficiente de amortiguamiento se estimaron a partir de la respuesta libre de la estructura a diferentes amplitudes utilizando 10 ciclos. En este intervalo, la frecuencia se obtiene como la inversa del periodo y el amortiguamiento a partir del decremento logarítmico. Los resultados obtenidos se muestran en la Figura 9, donde se observa que para todo el rango de aceleraciones, la frecuencia varía menos de un $1 \%$, lo cual se puede considerar despreciable a efectos de su consideración en los modelos de carga. Por otro lado, la variación del amortiguamiento es más significativa que en el caso de la frecuencia. Su comportamiento es el habitual, a mayores excitaciones mayores valores de amortiguamiento, aunque el valor se mantiene por debajo de $0.5 \%$ para las excitaciones esperadas (rara vez se superan los $2-3 \mathrm{~m} / \mathrm{s}^{2}$ ). En el caso de excitaciones de baja amplitud, la estimación del amortiguamiento es menos precisa y se tiene una mayor variabilidad en los valores de amortiguamiento debido a que el ruido y la respuesta de la estructura llegan a ser del mismo orden. Por todo ello, para el rango de excitaciones esperadas, se puede considerar que la estructura se comporta linealmente. Obsérvese además que se corroboraron los valores de frecuencia y amortiguamiento obtenidos mediante el AMO (ver Tabla 1).

\section{Estimación de los valores de respuesta}

En esta sección se describen algunos modelos de carga recogidos en normativas y guías de cálculo de pasarelas. Se realiza una descripción de los modelos, la metodología 
empleada y los valores obtenidos para la PMC utilizando el Eurocódigo 5 (8), la ISO 10137 (9) y la guía Setra (10). Las estimaciones se realizaron teniendo en cuenta las pruebas experimentales realizadas con objeto de comparar ambas. Se utilizaron 4 peatones con diferentes masas $(102,92,72$ y $63 \mathrm{~kg}$ ) y se realizaron varias pruebas con cada una de las combinaciones posibles de grupos de 1, 2, 3 y 4 peatones. También se realizaron pruebas con peatones distribuidos aleatoriamente (flujo de peatones) de 2 , 3,4 y 15 personas.

\subsection{Eurocódigo 5}

El Eurocódigo 5 (8) es una normativa referida al cálculo y comprobación de puentes de madera, aunque el modelo definido (en el Anejo B) no es específico para madera y por ello puede ser usado para evaluar puentes de cualquier material. Se supone que el puente se comporta como una viga simplemente apoyada y la aceleración máxima vertical bajo la carga de un peatón (modelada como una fuerza armónica estacionaria a la frecuencia natural del puente) se estima mediante la siguiente ecuación: [1]

[1] $a_{1}= \begin{cases}\frac{200}{M \zeta} & \text { para } f_{n} \leq 2,5 \mathrm{~Hz} \\ \frac{100}{M \zeta} & \text { para } 2,5 \mathrm{~Hz}<f_{n} \leq 5,0 \mathrm{~Hz}\end{cases}$

donde $M, \zeta$ y $f_{n}$ son la masa total del vano del puente, el coeficiente de amortiguamiento (referido al crítico) del modo de vibración considerado y la frecuencia natural de dicho modo, respectivamente. Para el modelado del primer armónico se ha utilizado una fuerza de amplitud constante de $200 \mathrm{~N}$ y para el segundo armónico de $100 \mathrm{~N}$. Estas fuerzas se obtienen bajo la hipótesis de que la fuerza dinámica de un peatón $(700 \mathrm{~N})$ es igual al $40 \%$ y al 20 $\%$ para el primer y el segundo armónico y multiplicado por 0,7 para tener en cuenta que no se alcanza el estado estacionario (34). En el caso de un peatón corriendo la aceleración viene definida por [2]

$$
a_{1}=\frac{600}{M \zeta} \text { para } 2,5 \mathrm{~Hz}<f_{n} \leq 5,0 \mathrm{~Hz}
$$

donde la fuerza dinámica es el 120 \% del peso del peatón.

$\mathrm{Si}$ se considera un flujo de $\mathrm{N}$ peatones presentes en el puente al mismo tiempo, la aceleración se obtiene como sigue [3]

$$
\text { [3] } \quad a_{n}=0,23 a_{1} N k
$$

donde $a_{1}$ es la aceleración generada por un peatón y el factor $k$ reduce el número de peatones sincronizados si la frecuencia natural del puente está alejada de las frecuencias habituales de paso (Figura 10).
Para calcular este factor $k$ se considera que la frecuencia normal al andar está entre 1,5 y $2,5 \mathrm{~Hz}$ y en ese intervalo existe una sincronización perfecta pero para frecuencias fuera de ese intervalo la sincronización es menos probable y el factor $\mathrm{k}$ disminuye. La norma indica que el número de peatones debe tomarse igual a 13 para grupos de personas e igual a $0,6 \mathrm{~A}$ para un flujo continuo (siendo $A$ el área del puente en $\mathrm{m}^{2}$ ).

Las limitaciones del cálculo de la aceleración aplicando este método son: (i) es aplicable únicamente a estructuras tipo viga y (ii) no tiene en cuenta que la amplitud de la fuerza es función de la frecuencia de paso.

En la Tabla 3 se muestran las aceleraciones máximas calculadas según la formulación presente en el Eurocódigo 5, utilizando los parámetros modales de la pasarela calculados inicialmente sin ocupación (parámetros iniciales (P. i.)) y los parámetros calculados teniendo en cuenta la influencia de los peatones presentes en la pasarela (parámetros corregidos (P. c.), (ver Tabla 2)). Se ha considerado que la influencia en los parámetros modales de la estructura de los peatones andando y corriendo es la misma a efectos de la estimación de la aceleración. Los valores mostrados para 1, 2, 3 y 4 peatones son los valores medios considerando los cuatro peatones por separado y todas las posibles combinaciones entre ellos. Tal y como era de esperar, se observa la gran importancia que tiene el amortiguamiento en la estimación de las aceleraciones.

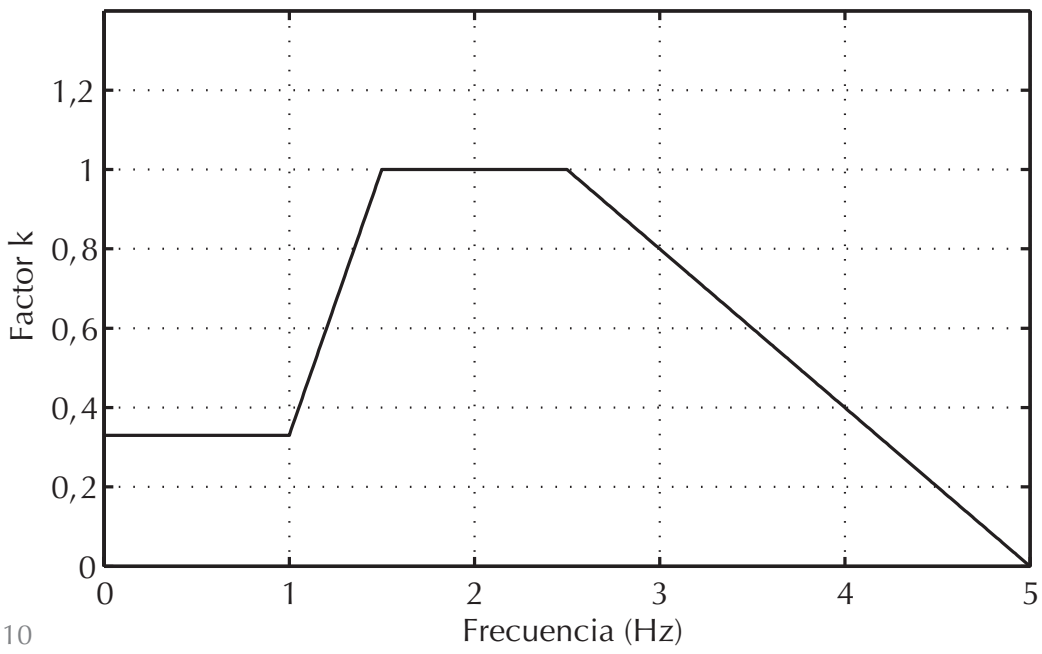

Tabla 3. Aceleraciones máximas $\left(\mathrm{m} / \mathrm{s}^{2}\right)$ de la pasarela estimadas según el Eurocódigo 5

\begin{tabular}{|c|c|c|c|c|}
\hline \multirow{2}{*}{ Peatones } & \multicolumn{2}{|c|}{ Andando } & \multicolumn{2}{c|}{ Corriendo } \\
\cline { 2 - 5 } & P. i. & P. c. & P. i. & P. c. \\
\hline 1 & 1,03 & 0,87 & 6,16 & 5,24 \\
\hline 2 & 1,72 & 1,06 & 10,98 & 6,76 \\
\hline 3 & 1,76 & 1,00 & 10,98 & 6,28 \\
\hline 4 & 1,83 & 1,02 & 12,01 & 6,67 \\
\hline 15 & 1.67 & 0.29 & - & - \\
\hline
\end{tabular}

10. Factor $k$ de sincronización según el Eurocódigo 5. 
11. Estimación de la respuesta de la estructura utilizando la fuerza de un peatón modulada por el modo de vibración.

12a. Modelo de fuerza para un peatón de $102 \mathrm{~kg}$ corriendo (amplitud constante y amplitud modulada)

12b. Respuesta estimada en la pasarela (con fuerza de amplitud constante y con fuerza de amplitud modulada

\subsection{ISO 10137}

En la norma ISO 10137 (9) se introducen recomendaciones para el cálculo del ELSV en edificios y puentes. Para el estudio de vibraciones inducidas por humanos se propone (Anejo A) un modelo de carga para la fuerza vertical (periódica) generada por un peatón de la forma [4]

[4]

$$
F_{1}(t)=Q\left(1+\sum_{n=1}^{k} \alpha_{n} \sin \left(2 \pi n f_{p} t+\varphi_{n}\right)\right)
$$

donde $Q$ es el peso del peatón, $\alpha_{n}$ es el factor dinámico de carga (FDC) que representa la proporción de la fuerza armónica al andar con respecto al peso, $\varphi_{n}$ el ángulo de fase de la carga armónica n-ésima, $f_{p}$ la frecuencia de paso y $k$ el número de armónicos que caracterizan la fuerza en el rango de frecuencias de interés. Para el caso de un peatón andando, el FDC del primer

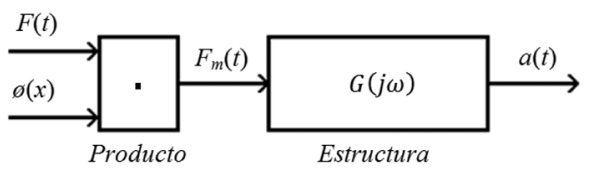

11

Fuerza de excitación

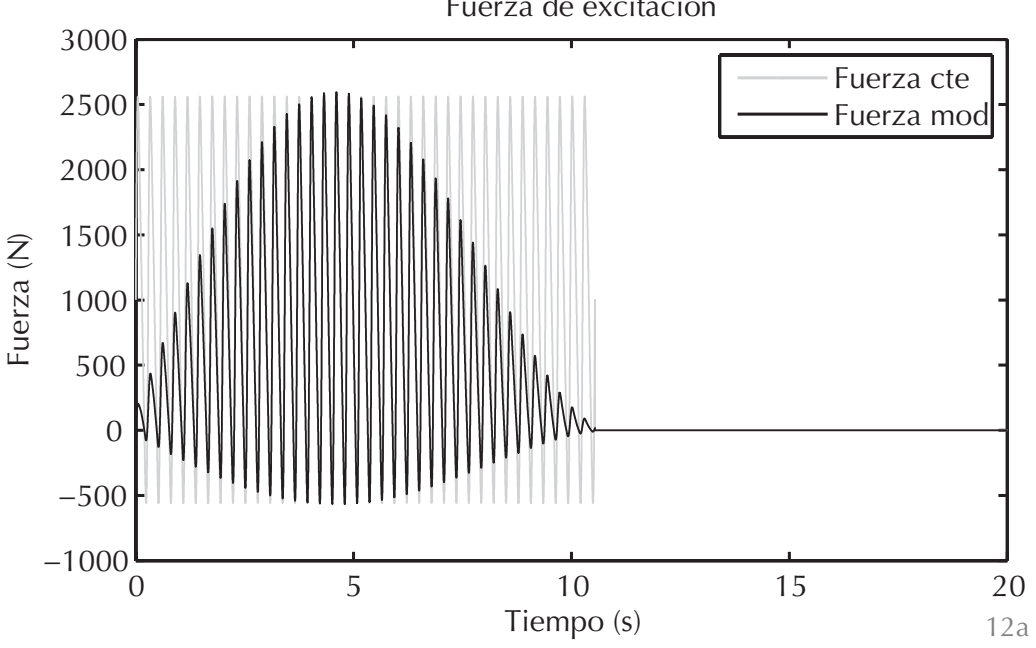

Respuesta de la pasarela

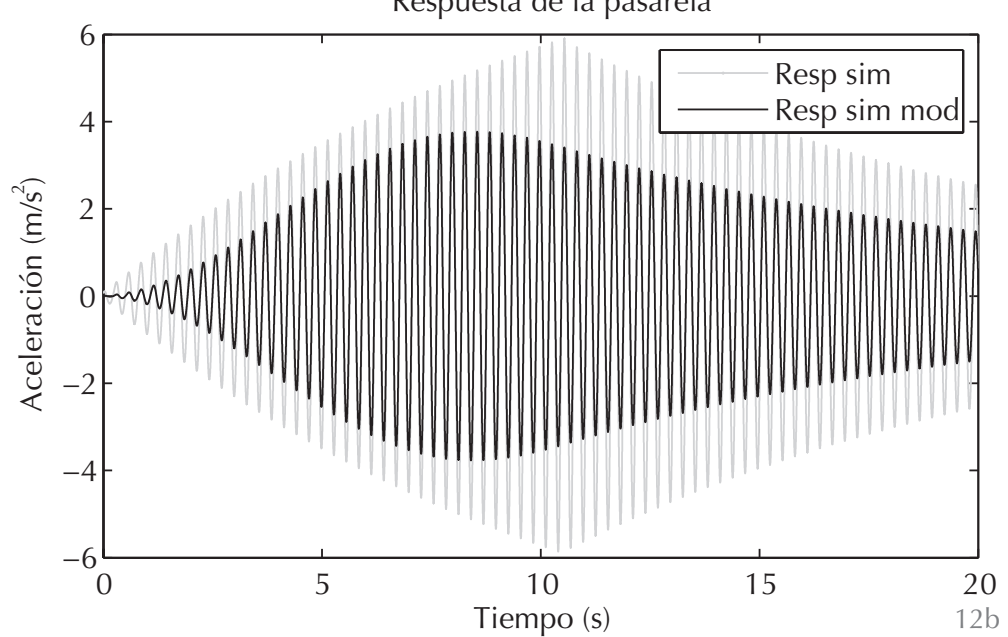

armónico es función de la frecuencia $f_{p}$ $\alpha_{1}=0,37\left(f_{p}-1\right)$, mientras que los armónicos mayores son independientes de la frecuencia de paso, $\alpha_{2}=0,1, \alpha_{3}=\alpha_{4}=\alpha_{5}=0,06$. Para calcular la carga de un peatón corriendo los FDC serían $\alpha_{1}=1,4, \alpha_{2}=0,4$ y $\alpha_{3}=0,1$. Para el caso de un grupo de $N$ peatones, la fuerza dinámica se multiplicaría por $\mathrm{N}$ si están sincronizados y por $\sqrt{N}$ si están desincronizados.

La norma ISO 10137, a diferencia del Eurocódigo 5, define el modelo de fuerza en lugar de definir directamente la respuesta. Esto hace que la estimación de la respuesta sea mejor ya que se puede usar un modelo acorde al tipo de estructura y no tener que suponer que es una estructura tipo viga. Para calcular la respuesta de aceleración de la estructura $(a(t))$ se introduce la fuerza $(F(t))$ en el modelo de la pasarela bien directamente o de forma modulada $\left(F_{m}(t)\right)$ considerando el modo de vibración $(\varnothing(x))$ escalado a 1 el máximo valor, donde $x$ es la coordenada longitudinal que se puede expresar como $x=v \cdot t$, siendo $v$ la velocidad del peatón y $t$ el tiempo (Figura 11). La estructura se ha modelado considerando un sistema de un grado de libertad, siendo la función de transferencia entre la aceleración en el centro del vano y la fuerza en ese punto [5]

$$
G(j \omega)=\frac{-\frac{1}{m_{1}} \omega^{2}}{\omega^{2}-\omega_{1}^{2}+j 2 \zeta_{1} \omega_{1} \omega}
$$

donde $\omega$ es la frecuencia en $\mathrm{rad} / \mathrm{s}$ y $\zeta_{1}, \omega_{1}$ y $m_{1}$ son el amortiguamiento, la frecuencia natural y la masa modal asociada al primer modo, respectivamente.

Se calcula la fuerza generada según cada caso de carga y se introduce en el modelo para calcular la aceleración máxima en el punto central. En la Figura 12 se muestra un ejemplo del resultado estimado para el caso de un peatón de $102 \mathrm{~kg}$ corriendo a la primera frecuencia de resonancia de la pasarela $(3,5 \mathrm{~Hz})$ y a una velocidad de $5 \mathrm{~m} / \mathrm{s}$.

En la Tabla 4 se muestran las aceleraciones máximas calculadas según la formulación presente en la norma ISO 10137 utilizando la fuerza constante (F. c.) y la fuerza modulada (F. m.) por el modo de vibración para peatones desincronizados y sincronizados. Obsérvese que para peatones sincronizados se obtienen valores estimados bastante mayores que para desincronizados. También se utilizan los parámetros modales de la pasarela calculados inicialmente sin ocupación (P. i.) y los parámetros calculados teniendo en cuenta la influencia de los peatones presentes en la pasarela (P. c.) (ver Tabla 2). 


\subsection{Guía de pasarelas SETRA}

En la guía de cálculo publicada por el Ministerio de Transportes e Infraestructuras francés (10) se presenta un modelo de carga que considera la carga del peatón (andando y corriendo) como una fuerza puntual aplicada sobre el tablero función del tiempo y de la posición del peatón. Teniendo en cuenta que $x$ es la posición del peatón con respecto a la pasarela, la carga de un peatón moviéndose a velocidad constante $\mathrm{v}$ puede ser representada por el producto de una componente temporal $F(t)$ y una componente espacial $\delta(x-v \cdot t)$, siendo $\delta$ la delta de Dirac [6].

$$
P(x, t)=F(t) \delta(x-v t)
$$

La fuerza ejercida $F(t)$ se descompone, al igual que en la ISO 10137, mediante series de Fourier como sigue[7]

$$
\begin{aligned}
& F(t)=G_{0}+G_{1} \sin 2 \pi f_{m} t+ \\
& +\sum_{i=2}^{n} G_{i} \sin \pi\left(2 \pi i f_{m} t+\varphi_{i}\right)
\end{aligned}
$$

donde $G_{0}$ es el peso del peatón, $G_{1}$ es la amplitud del primer armónico, $G_{i}$ la amplitud del armónico i, $f_{m}$ la frecuencia de paso, $\varphi_{i}$ el ángulo de fase del armónico i con respecto al primero y $\mathrm{n}$ el número de armónicos tenidos en cuenta. Los valores de los coeficientes de la serie de Fourier para el caso de un peatón andando, teniendo en cuenta los primeros tres términos al considerar los demás despreciables, son: $G_{1}=0,4 G_{0}\left(0,5 G_{0}\right.$ si $f_{m}=2,4 \mathrm{~Hz}$ y $0,1 G_{0}$ si $\left.f_{m}=1 \mathrm{~Hz}\right), G_{2}=G_{3}=0,1 G_{0}, \varphi_{2}=\varphi_{3}=\pi / 2$.

Para el caso de un peatón corriendo, la guía establece que la componente vertical de la carga puede aproximarse por una secuencia de semisenoides representada, después de una serie de simplificaciones, como sigue [8]

$$
\text { [8] }
$$

$F(t)=\left\{\begin{array}{cc}G_{0}+\sum_{i=1}^{n} G_{i} \sin 2 \pi i f_{m} t & \text { para }(j-1) T_{m} \leq t \leq(j-1 / 2) T_{m} \\ 0 & \text { para }(j-1 / 2) T_{m}<t \leq j T_{m}\end{array}\right.$

donde $G_{0}$ es el peso del peatón, $G_{1}=1,6 G_{0}$, $G_{2}=0,7 G_{0}, G_{3}=0,2 G_{0}, f_{m}$ la frecuencia de paso, $T_{m}$ el periodo $\left(T_{m}=1 / f_{m}\right)$ y j el número de paso $(j=1,2$, etc.).

La guía establece un modelo de carga para flujo de peatones que consiste en cargas armónicas distribuidas que causen aceleraciones iguales al 95\% de la aceleración pico debido a flujos de peatones aleatorios. La amplitud de la carga distribuida se determina transformando el número de peatones aleatorio en un número equivalente

\begin{tabular}{|c|c|c|c|c|c|c|c|c|}
\hline \multirow{4}{*}{ Peatones } & \multicolumn{8}{|c|}{ Desincronizados } \\
\hline & \multicolumn{4}{|c|}{ Andando } & \multicolumn{4}{|c|}{ Corriendo } \\
\hline & \multicolumn{2}{|c|}{ P. i. } & \multicolumn{2}{|c|}{ P. c. } & \multicolumn{2}{|c|}{ P. i. } & \multicolumn{2}{|c|}{ P. c. } \\
\hline & F. c. & F. $m$. & F. c. & F. $m$. & F. c. & F. m. & F. c. & F. m. \\
\hline 1 & - & - & - & - & - & - & - & - \\
\hline 2 & 0,62 & 0,56 & 0,42 & 0,40 & 9,51 & 6,75 & 6,52 & 5,10 \\
\hline 3 & 0,78 & 0,70 & 0,50 & 0,47 & 11,40 & 8,00 & 7,42 & 5,82 \\
\hline 4 & 0,94 & 0,87 & 0,58 & 0,56 & 14,07 & 9,66 & 9,10 & 7,03 \\
\hline \multirow{4}{*}{ Peatones } & \multicolumn{8}{|c|}{ Sincronizados } \\
\hline & \multicolumn{4}{|c|}{ Andando } & \multicolumn{4}{|c|}{ Corriendo } \\
\hline & \multicolumn{2}{|c|}{ P. i. } & \multicolumn{2}{|c|}{ P. c. } & \multicolumn{2}{|c|}{ P. i. } & \multicolumn{2}{|c|}{ P. c. } \\
\hline & F. c. & F. m. & F. c. & F. $\mathrm{m}$. & F. c. & F. $\mathrm{m}$. & F. c. & F. $\mathrm{m}$. \\
\hline 1 & 0,47 & 0,42 & 0,42 & 0,38 & 6,37 & 4,56 & 5,68 & 4,18 \\
\hline 2 & 0,89 & 0,79 & 0,60 & 0,56 & 13,45 & 9,55 & 9,21 & 7,22 \\
\hline 3 & 1,36 & 1,21 & 0,86 & 0,81 & 19,75 & 13,85 & 12,85 & 10,08 \\
\hline 4 & 1,88 & 1,74 & 1,16 & 1,13 & 28,14 & 19,32 & 18,19 & 14,05 \\
\hline
\end{tabular}
de peatones que caminan a la frecuencia natural y se distribuyen uniformemente en
Tabla 4. Aceleraciones máximas $\left(\mathrm{m} / \mathrm{s}^{2}\right)$ de la pasarela estimadas según la ISO 10137

la longitud del puente. Este número se ha calculado empíricamente obteniendo la respuesta estructural de diversos puentes con diferentes longitudes, amortiguamientos y modos de vibración sometidos a flujos de peatones de diferentes densidades, y se define como [9]

[9]

$n_{\text {eff }}= \begin{cases}10,8 \sqrt{N \zeta} & \text { para } d \leq 0,8 \text { peatones } / \mathrm{m}^{2} \\ 1,85 \sqrt{N} & \text { para } d \geq 1,0 \text { peatón } / \mathrm{m}^{2}\end{cases}$

donde $N$ es el número de peatones, $\zeta$ el amortiguamiento y $d$ es la densidad de peatones presentes en la pasarela.

En el modelo se supone que la distribución de la frecuencia de paso de los peatones sigue una distribución gaussiana. La fuerza dinámica por unidad de área queda definida así [10]

[10]

$$
f_{N}(t)=\frac{F_{0} n_{\text {eff }}}{A} \psi \cos \left(2 \pi f_{n} t\right)
$$

donde $f_{0}$ es la amplitud de la carga de un único peatón (280 N para el primer armónico y $70 \mathrm{~N}$ para el segundo), A es el área del tablero del puente, $f_{m}$ la frecuencia natural y $\Psi \in[0,1]$ un factor que reduce la carga para frecuencias alejadas de la frecuencia normal de paso (Figura 13). Este factor $\Psi$ es análogo al factor $k$ del Eurocódigo 5.

La guía SETRA establece un caso de carga acorde a la ecuación 10 para cada modo de vibración con frecuencia por debajo de 5 $\mathrm{Hz}$ aplicada al tablero a la correspondiente frecuencia del modo de vibración. La dirección de la carga debe definirse según el signo del modo de vibración para considerar el escenario de carga más desfavorable. Además, el modelo de carga sólo incluye un armónico a la vez, por lo que la excitación multiarmónica de dos o más modos no se considera. Sin embargo, esto no es preocupante salvo para puentes singulares que pudieran tener los modos de vibración con frecuencias naturales cercanas. 


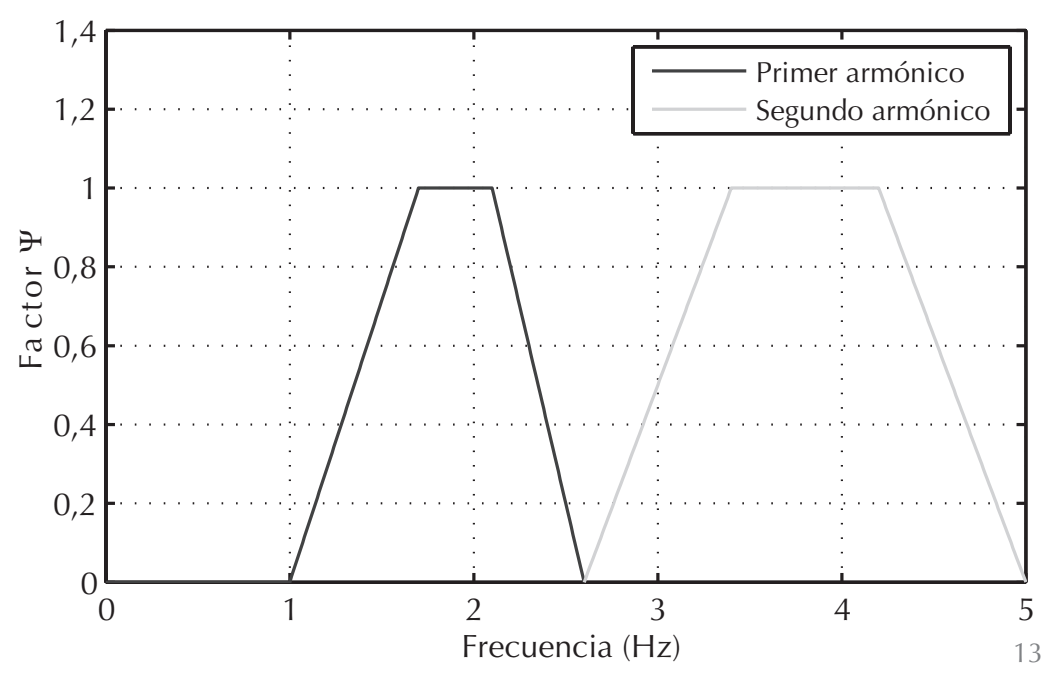

13. Factor $\Psi$ de sincronización según la guía SETRA

14. Pruebas en la pasarela peatonal.

Tabla 5. Aceleraciones máximas $\left(\mathrm{m} / \mathrm{s}^{2}\right)$ de la pasarela estimadas según la guía SETRA

\begin{tabular}{|c|c|c|c|c|c|c|c|c|}
\hline \multirow{3}{*}{ Peatones } & \multicolumn{4}{|c|}{ Andando } & \multicolumn{3}{c|}{ Corriendo } \\
\cline { 2 - 9 } & \multicolumn{2}{|c|}{ P. i. } & \multicolumn{2}{c|}{ P. c. } & \multicolumn{2}{c|}{ P. i. } & \multicolumn{2}{c|}{ P. c. } \\
\cline { 2 - 9 } & F. c. & F. m. & F. c. & F. m. & F. c. & F. m. & F. c. & F. m. \\
\hline $\mathbf{1}$ & 0,47 & 0,42 & 0,42 & 0,38 & 6,62 & 4,74 & 5,90 & 4,35 \\
\hline $\mathbf{2}$ & 0,59 & 0,35 & 0,46 & 0,28 & - & - & - & - \\
\hline $\mathbf{3}$ & 0,72 & 0,43 & 0,55 & 0,33 & - & - & - & - \\
\hline $\mathbf{4}$ & 0,83 & 0,50 & 0,62 & 0,37 & - & - & - & - \\
\hline $\mathbf{1 5}$ & 1,35 & 0,81 & 0,56 & 0,34 & - & - & - & - \\
\hline
\end{tabular}

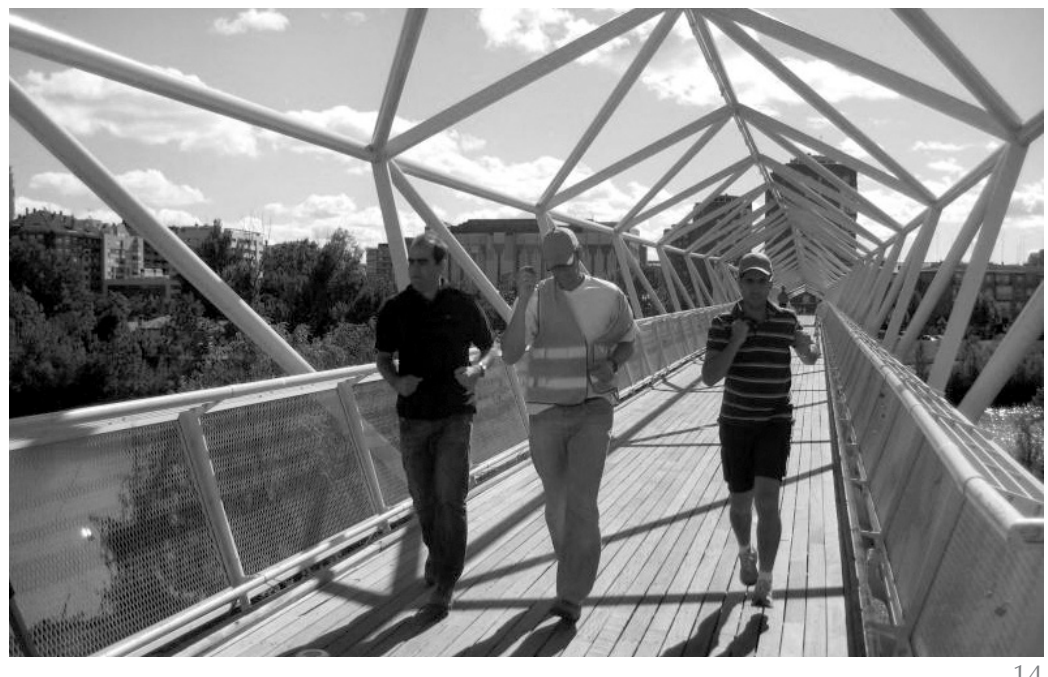

\section{PRUEBAS EXPERIMENTALES Y COMPARACIÓN CON LAS ESTIMACIONES}

En esta sección se presentan las pruebas realizadas y se comparan los resultados experimentales con los valores estimados.
Se realizaron pruebas con peatones andando, con peatones corriendo y con flujo continuo de peatones. Se utilizó un acelerómetro piezoeléctrico (como los usados en el AMO) colocado en el centro del vano para obtener la respuesta de la pasarela y se obtuvo el valor máximo y se comparó con los valores estimados. La Figura 14 muestra una de las pruebas realizadas en la pasarela, un grupo de tres peatones corriendo a la frecuencia natural de la pasarela $(3,5 \mathrm{~Hz})$.

\subsection{Peatones andando}

El peatón o el grupo de peatones recorren el vano 2 andando de un extremo al otro a la frecuencia de $1,75 \mathrm{~Hz}$ para excitar el primer modo de la pasarela con el segundo armónico de la excitación. Se utilizaron 4 sujetos diferentes para las pruebas y se emplearon grupos de 1, 2, 3 y 4 peatones. Los peatones intentaban caminar de forma sincronizada (con la ayuda de un metrónomo) y se grabaron las pruebas en video para estimar la velocidad con la que los peatones cruzaban la pasarela, parámetro necesario en las simulaciones. Se realizaron varias pruebas con cada configuración y se compararon los resultados experimentales con los obtenidos usando las guías de cálculo. La Tabla 6 muestra los resultados experimentales obtenidos y su comparación con los estimados (Tablas 3, 4 y 5). La guía SETRA no considera grupos de peatones, sólo un peatón o flujo de peatones. A modo de ejemplo, la comparación de la respuesta experimental de la pasarela con la respuesta estimada según la norma ISO 10137 provocada por un peatón andando a la frecuencia de paso de $1,75 \mathrm{~Hz}$ y a una velocidad de $1 \mathrm{~m} / \mathrm{s}$ se muestra en la Figura 15.

\subsection{Peatones corriendo}

Un corredor o un grupo de corredores recorren el vano 2 de un extremo al otro a la frecuencia de $3,5 \mathrm{~Hz}$ para excitar el primer modo de la pasarela. Se utilizaron 4 sujetos diferentes para las pruebas y se emplearon grupos de 1, 2, 3 y 4 corredores. Los peatones intentaban correr de forma sincronizada y se realizaron varias pruebas con cada configuración, comparándose los resultados experimentales con los estimados. La Tabla 7 muestra los resultados experimentales obtenidos y su comparación con los estimados. La guía SETRA no considera grupos de corredores. A modo de ejemplo, la comparación de la respuesta experimental de la pasarela con la respuesta simulada según la norma ISO 10137 provocada por un corredor a una frecuencia de paso de $3,5 \mathrm{~Hz}$ y a una velocidad de $5 \mathrm{~m} / \mathrm{s}$ se muestra en la Figura 16. 
Tabla 6. Aceleraciones experimentales máximas $\left(\mathrm{m} / \mathrm{s}^{2}\right)$ de la pasarela, aceleraciones máximas estimadas $\left(\mathrm{m} / \mathrm{s}^{2}\right)$ y variación con respecto a los valores experimentales $(\%)$

\begin{tabular}{|c|c|c|c|c|c|c|c|c|c|}
\hline \multirow{3}{*}{$\begin{array}{c}\text { Peatones } \\
1\end{array}$} & \multirow{3}{*}{$\begin{array}{c}\text { Exper. } \\
0,40\end{array}$} & \multicolumn{8}{|c|}{ Eurocódigo 5} \\
\hline & & \multicolumn{4}{|c|}{ P. i. } & \multicolumn{4}{|c|}{ P. c. } \\
\hline & & \multicolumn{2}{|c|}{1,03} & \multicolumn{2}{|c|}{$157,72 \%$} & \multicolumn{2}{|c|}{0,87} & \multicolumn{2}{|c|}{$116,78 \%$} \\
\hline 2 & 0,41 & \multicolumn{2}{|c|}{1,72} & \multicolumn{2}{|c|}{$319,19 \%$} & \multicolumn{2}{|c|}{1,06} & \multicolumn{2}{|c|}{$157,96 \%$} \\
\hline 3 & 0,61 & \multicolumn{2}{|c|}{1,76} & \multicolumn{2}{|c|}{$186,46 \%$} & \multirow{2}{*}{\multicolumn{2}{|c|}{$\begin{array}{l}\frac{1,00}{1,02} \\
\end{array}$}} & \multicolumn{2}{|c|}{$63,69 \%$} \\
\hline 4 & 0,63 & \multicolumn{2}{|c|}{1,83} & \multicolumn{2}{|c|}{$192,09 \%$} & & & \multicolumn{2}{|c|}{$62,28 \%$} \\
\hline \multirow{4}{*}{ Peatones } & \multirow{4}{*}{ Exper. } & \multicolumn{8}{|c|}{ ISO 10137} \\
\hline & & \multicolumn{8}{|c|}{ Desincronizados } \\
\hline & & \multicolumn{4}{|c|}{ P. i. } & \multicolumn{4}{|c|}{ P. c. } \\
\hline & & \multicolumn{2}{|c|}{ F. c. } & \multicolumn{2}{|c|}{ F. $m$. } & \multicolumn{2}{|c|}{ F. c. } & \multicolumn{2}{|c|}{ F. m. } \\
\hline 1 & 0,40 & & - & & - & & - & & - \\
\hline 2 & 0,41 & 0,62 & $52,23 \%$ & 0,56 & $36,12 \%$ & 0,42 & $3,08 \%$ & 0,40 & $-3,32 \%$ \\
\hline 3 & 0,61 & 0,78 & $27,41 \%$ & 0,70 & $14,15 \%$ & 0,50 & $-19,08 \%$ & 0,47 & $-23,53 \%$ \\
\hline 4 & 0,63 & 0,94 & $50,00 \%$ & 0,87 & $39,17 \%$ & 0,58 & $-7,04 \%$ & 0,56 & $-10,20 \%$ \\
\hline \multirow{3}{*}{ Peatones } & \multirow{3}{*}{ Exper. } & & & & Sincro & izados & & & \\
\hline & & & & & & & & & \\
\hline & & & c. c. & & $\mathrm{m}$. & & c. & & $\mathrm{m}$. \\
\hline 1 & 0,40 & 0,47 & $16,55 \%$ & 0,42 & $4,24 \%$ & 0,42 & $3,03 \%$ & 0,38 & $-6,11 \%$ \\
\hline 2 & 0,41 & 0,88 & $115,28 \%$ & 0,79 & $92,51 \%$ & 0,60 & $45,78 \%$ & 0,56 & $36,73 \%$ \\
\hline 3 & 0,61 & 1,35 & $120,68 \%$ & 1,21 & $97,71 \%$ & 0,86 & $40,16 \%$ & 0,81 & $32,46 \%$ \\
\hline 4 & 0,63 & 1,88 & $200,01 \%$ & 1,78 & $178,34 \%$ & 1,16 & $85,91 \%$ & 1,13 & $79,59 \%$ \\
\hline & & & & & & & & & \\
\hline Peatones & Exper. & & & & & & & & \\
\hline & & & .c. & & $\mathrm{m}$. & & c. & & $\mathrm{m}$. \\
\hline 1 & 0,40 & 0,47 & $17,18 \%$ & 0,42 & $4,69 \%$ & 0,42 & $3,53 \%$ & 0,38 & $5,90 \%$ \\
\hline
\end{tabular}

Tabla 7. Aceleraciones experimentales máximas $\left(\mathrm{m} / \mathrm{s}^{2}\right)$ de la pasarela, aceleraciones máximas estimadas $\left(\mathrm{m} / \mathrm{s}^{2}\right)$ y variación con respecto a los valores experimentales $(\%)$

\begin{tabular}{|c|c|c|c|c|c|c|c|c|c|}
\hline \multirow{3}{*}{$\begin{array}{c}\text { Corredores } \\
1\end{array}$} & \multirow{3}{*}{$\begin{array}{c}\text { Exper. } \\
3,72\end{array}$} & \multicolumn{8}{|c|}{ Eurocódigo 5} \\
\hline & & \multicolumn{4}{|c|}{ P. i. } & \multicolumn{4}{|c|}{ P. c. } \\
\hline & & \multicolumn{2}{|c|}{6,16} & \multicolumn{2}{|c|}{$65,78 \%$} & \multicolumn{2}{|c|}{5,24} & \multicolumn{2}{|c|}{$41,15 \%$} \\
\hline 2 & 6,49 & \multicolumn{2}{|c|}{10,98} & \multicolumn{2}{|c|}{$69,30 \%$} & \multicolumn{2}{|c|}{6,76} & \multicolumn{2}{|c|}{$4,18 \%$} \\
\hline 3 & 8,01 & \multicolumn{2}{|c|}{10,98} & \multicolumn{2}{|c|}{$37,07 \%$} & \multicolumn{2}{|c|}{6,28} & \multicolumn{2}{|c|}{$-21,67 \%$} \\
\hline 4 & 8,55 & \multicolumn{2}{|c|}{12,01} & \multicolumn{2}{|c|}{$40,58 \%$} & \multicolumn{2}{|c|}{6,67} & \multicolumn{2}{|c|}{$-21,90 \%$} \\
\hline \multirow{4}{*}{ Corredores } & \multirow{4}{*}{ Exper. } & \multicolumn{8}{|c|}{ ISO 10137} \\
\hline & & \multicolumn{8}{|c|}{ Desincronizados } \\
\hline & & \multicolumn{4}{|c|}{ P. i. } & \multicolumn{4}{|c|}{ P. c. } \\
\hline & & \multicolumn{2}{|c|}{ F. c. } & \multicolumn{2}{|c|}{ F. $m$. } & \multicolumn{2}{|c|}{ F. c. } & \multicolumn{2}{|c|}{ F. m. } \\
\hline 1 & 3,72 & & - & & - & & - & & - \\
\hline 2 & 6,49 & 9,51 & $46,64 \%$ & 6,75 & $4,07 \%$ & 6,52 & $0,46 \%$ & 5,10 & $-21,32 \%$ \\
\hline 3 & 8,01 & 11,40 & $42,31 \%$ & 8,00 & $-0,19 \%$ & 7,42 & $-7,41 \%$ & 5,82 & $-27,37 \%$ \\
\hline 4 & 8,55 & 14,07 & $64,62 \%$ & 9,66 & $13,04 \%$ & 9,10 & $6,44 \%$ & 7,03 & $-17,79 \%$ \\
\hline \multirow{3}{*}{ Corredores } & & & & & Sincro & izados & & & \\
\hline & Exper. & & & & & & & & \\
\hline & & & F. c. & & $\mathrm{m}$. & & c. & & $\mathrm{m}$. \\
\hline 1 & 3,72 & 6,37 & $71,34 \%$ & 4,56 & $22,64 \%$ & 5,68 & $52,83 \%$ & 4,18 & $12,50 \%$ \\
\hline 2 & 6,49 & 13,45 & $107,38 \%$ & 9,55 & $47,17 \%$ & 9,21 & $42,07 \%$ & 7,22 & $11,27 \%$ \\
\hline 3 & 8,01 & 19,75 & $146,49 \%$ & 13,85 & $72,88 \%$ & 12,85 & $60,36 \%$ & 10,08 & $25,80 \%$ \\
\hline 4 & 8,55 & 28,14 & $229,24 \%$ & 19,32 & $126,08 \%$ & 18,19 & $112,89 \%$ & 14,05 & $64,42 \%$ \\
\hline & & & & & SEI & $\mathrm{RA}$ & & & \\
\hline Corredores & Exper. & & & & & & & c. & \\
\hline & & & F. c. & & $\mathrm{m}$ & & c. & & $\mathrm{m}$. \\
\hline 1 & 3,72 & 6,62 & $78,12 \%$ & 4,74 & $27,69 \%$ & 5,90 & $58,73 \%$ & 4,35 & $17,00 \%$ \\
\hline
\end{tabular}

15. Respuesta de la pasarela peatonal excitada por un peatón andando (estimada usando fuerza de amplitud constante, estimada usando fuerza de amplitud modulada y experimental).

16. Respuesta de la pasarela peatonal excitada por un corredor (estimada usando fuerza de amplitud constante, estimada usando fuerza de amplitud modulada y experimental).
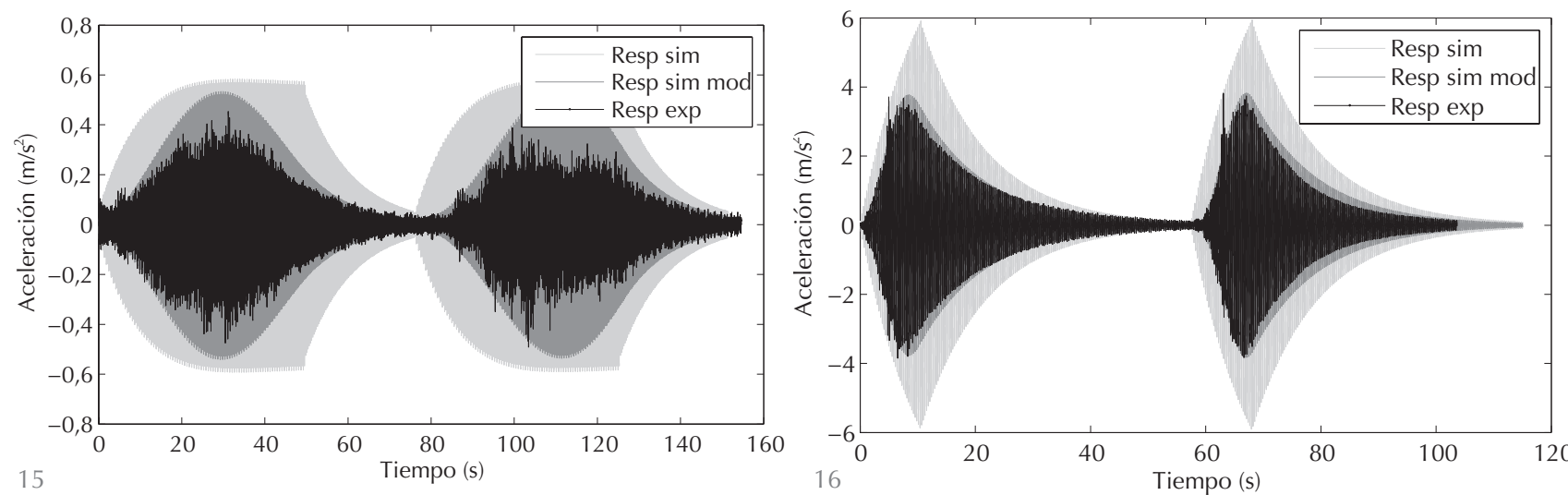
17. Respuesta de la pasarela peatonal excitada por un flujo continuo de 15 peatones (estimada usando fuerza constante, estimada usando fuerza modulada y experimental).

Tabla 8. Aceleraciones experimentales máximas $\left(\mathrm{m} / \mathrm{s}^{2}\right)$ de la pasarela, aceleraciones máximas estimadas $\left(\mathrm{m} / \mathrm{s}^{2}\right)$ y variación con respecto a los valores experimentales $(\%)$

\begin{tabular}{|c|c|c|c|c|c|c|c|c|c|}
\hline \multirow{3}{*}{$\begin{array}{c}\text { Peatones } \\
2 \\
\end{array}$} & \multirow{3}{*}{$\begin{array}{c}\text { Exper. } \\
0,42 \\
\end{array}$} & \multicolumn{8}{|c|}{ Eurocódigo 5} \\
\hline & & \multicolumn{4}{|c|}{ P. i. } & \multicolumn{4}{|c|}{ P. c. } \\
\hline & & \multicolumn{2}{|c|}{2,00} & \multicolumn{2}{|c|}{$378,04 \%$} & \multicolumn{2}{|c|}{1,23} & \multicolumn{2}{|c|}{$194,18 \%$} \\
\hline 3 & 0,46 & \multicolumn{2}{|c|}{2,00} & \multicolumn{2}{|c|}{$338,86 \%$} & \multicolumn{2}{|c|}{1,14} & \multicolumn{2}{|c|}{$150,78 \%$} \\
\hline 4 & 0,50 & \multicolumn{2}{|r|}{2,00} & \multicolumn{2}{|c|}{$296,61 \%$} & \multicolumn{2}{|c|}{1,11} & \multicolumn{2}{|c|}{$120,34 \%$} \\
\hline 15 & 0,56 & & 1,67 & \multicolumn{2}{|c|}{$200,02 \%$} & \multicolumn{2}{|c|}{0,29} & \multicolumn{2}{|c|}{$-48,44 \%$} \\
\hline \multirow{3}{*}{ Peatones } & \multirow{3}{*}{ Exper. } & \multicolumn{8}{|c|}{ SETRA } \\
\hline & & \multicolumn{4}{|c|}{ P. i. } & \multicolumn{4}{|c|}{ P. c. } \\
\hline & & \multicolumn{2}{|r|}{ F. c. } & \multicolumn{2}{|c|}{ F. m. } & \multicolumn{2}{|c|}{ F. c. } & \multicolumn{2}{|c|}{ F. $m$. } \\
\hline 2 & 0,42 & 0,59 & $40,82 \%$ & 0,35 & $-15,37 \%$ & 0,46 & $10,46 \%$ & 0,28 & $-33,61 \%$ \\
\hline 3 & 0,46 & 0,72 & $58,32 \%$ & 0,43 & $-4,87 \%$ & 0,55 & $19,68 \%$ & 0,33 & $-28,07 \%$ \\
\hline 4 & 0,50 & 0,83 & $65,22 \%$ & 0,50 & $-0,71 \%$ & 0,62 & $23,15 \%$ & 0,37 & $-26,01 \%$ \\
\hline 15 & 0,56 & 1,35 & $142,04 \%$ & 0,81 & $45,45 \%$ & 0,56 & $0,34 \%$ & 0,33 & $-39,70 \%$ \\
\hline
\end{tabular}

\subsection{Flujo continuo de peatones}

El ensayo consistió en hacer circular a un cierto número de peatones (2, 3, 4 y 15$)$ de forma continua durante 30 minutos, cada una a su ritmo. Se realizaron varias pruebas con cada configuración y se compararon los resultados experimentales con los estimados. La Tabla 8 muestra los resultados experimentales y su comparación con los estimados. La norma ISO 10137 no considera flujo de peatones. A modo de ejemplo, la comparación de la respuesta experimental de la pasarela con la respuesta estimada utilizando la guía SETRA provocada por un flujo continuo de 15 peatones se muestra en la Figura 17.

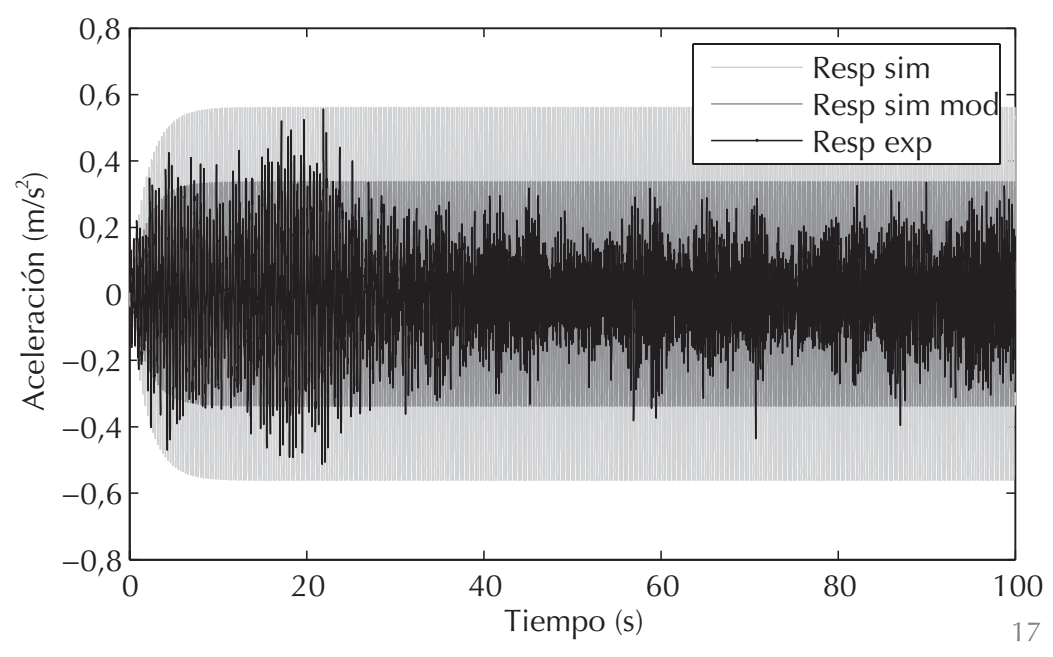

\section{CONCLUSIONES}

En este trabajo se han realizado estimaciones de la respuesta de una pasarela peatonal en servicio utilizando modelos de carga recogidos en guías y normativas de cálculo y se han comparado los resultados estimados con resultados experimentales obtenidos mediante la realización de numerosas pruebas.

Los resultados simulados y los registros experimentales indican que las guías de cálculo tienden a sobrestimar la respuesta de la estructura, obteniéndose para esta pasarela peatonal de media para todas las pruebas sobrestimaciones del $190 \%$ para el Eurocódigo 5, del 125\% para la ISO 10137 y del $70 \%$ para la SETRA. Si se tiene en cuenta la influencia de los peatones en los parámetros modales la estimación mejora, aunque se sigue sobrestimando el resultado en el Eurocódigo 5 (70\%) y en la ISO $10137(32 \%)$, no así en la SETRA (-17\%). De forma general para esta estructura las estimaciones para peatones andando (65\% media de todas las pruebas) dan peores resultados que las de peatones corriendo $(38 \%)$.

Se ha visto que la influencia de los peatones en las propiedades modales puede ser importante en la dirección vertical. Por tanto, sería recomendable que futuras guías de cálculo incluyan, de alguna manera, esta influencia evitando sobrestimaciones muy elevadas. Sin embargo, antes de que esto pueda ocurrir es necesario realizar cuantificaciones en numerosas pasarelas en servicio y realizar análisis estadísticos detallados.

Las pruebas se han realizado intentando que los peatones se muevan sincronizadamente. Los resultados muestran la dificultad de esta sincronización, aumentando la dificultad, como es lógico, cuanto mayor es el número de peatones que intentan sincronizarse. También se ha visto que la sincronización al andar es más complicada que al correr, motivo por el que las estimaciones andando dan peores resultados que corriendo, al tener valores experimentales menores que si estuvieran totalmente sincronizados.

\section{AGRADECIMIENTOS}

Este trabajo ha sido realizado en parte con la financiación aportada al proyecto de investigación BIA2011-28493 por el 
programa de apoyo a Proyectos de Investigación Fundamental No Orientada (PIFNO) del Ministerio de Ciencia e Innovación.
Los autores agradecen al Ayuntamiento de Valladolid y al Museo de la Ciencia su apoyo en las pruebas de campo.

\section{REFERENCIAS}

(1) Murcia, J. (2007). Tecnología de pasarelas con estructura de membrana. Informes de la Construcción, 59(507): 21-31, doi:10.3989/ic.2007.v59.i507.

(2) Jurado, J. A., Romera, L., Baldomir, A., Hernández, S.,Kusano, I. (2011). Aeroelastic analyses of a stress ribbon pedestrian bridge in Cuenca (Spain). Proc. 13th Int. Conf. on Wind Engineering, Amsterdam, The Netherlands.

(3) Zivanovic, S., Pavic, A., Ingolfsson, E. T. (2010). Modeling spatially unrestricted pedestrian traffic on footbridges. Journal of Structural Engineering, 136(10): 1296-1308.

(4) Bachmann H., Ammann W. (1987). Vibrations in structures induced by man and machines. Structural Engineering Documents 3e, IABSE.

(5) Sachse, R. (2002). The influence of human occupants on the dynamic properties of slender structures (PhD Thesis). University of Sheffield, UK.

(6) Zivanovic, S., Diaz, I. M., Pavic, A. (2009). Influence of walking and standing crowds on structural dynamic properties. Proc. 27th International Modal Analysis Conference IMAC XXVII, Orlando, USA.

(7) Máca, J., Valášek, M. (2011). Interaction of Human Gait and Footbridges. Proc. 8th International Conference on Structural Dynamics, EURODYN, Leuven, Belgium.

(8) UNE-EN 1995-2:2010. Eurocódigo 5: Proyecto de estructuras de madera. Parte 2: Puentes. AENOR, 2010.

(9) International Standard ISO 10137: 2007(E), Bases of for design of structures - Serviceability of buildings and walkways against vibrations. ISO 10137:2007, International Organization for Standardization, 2007.

(10) SETRA (2006). Technical guide - footbridges - Assessment of vibrational behavior of footbridges under pedestrian loading, Service d'Etudes Techniques des Routes et Autoroutes.

(11) RPM 95. (2003). Recomendaciones para el proyecto de puentes metálicos para carreteras. 2. ${ }^{a}$ reimpresión. Centro de Publicaciones Secretaría General Técnica Ministerio de Fomento, Madrid.

(12) RPX 95. (2003). Recomendaciones para el proyecto de puentes mixtos para carreteras. 2. reimpresión. Centro de Publicaciones Secretaría General Técnica Ministerio de Fomento, Madrid.

(13) IAP 98. (2003). Instrucción sobre acciones a considerar en el proyecto de puentes de carretera. 3. ${ }^{a}$ reimpresión. Centro de Publicaciones Secretaría General Técnica Ministerio de Fomento, Madrid.

(14) IAP 11. (2011). Instrucción sobre acciones a considerar en el proyecto de puentes de carretera. Centro de Publicaciones Secretaría General Técnica Ministerio de Fomento, Madrid.

(15) (1999). Recomendaciones para la realización de pruebas de carga de recepción en puentes de carretera. Centro de Publicaciones Secretaría General Técnica Ministerio de Fomento, Madrid.

(16) EHE-08. (2008). Instrucción de hormigón estructural. Centro de Publicaciones Secretaría General Técnica Ministerio de Fomento, Madrid.

(17) EAE. (2011). Instrucción de Acero Estructural. Centro de Publicaciones Secretaría General Técnica Ministerio de Fomento, Madrid.

(18) About the Eurocodes. Legal situation per country (latest update: 25/01/2011) http:// www.eurocodes-online.com.

(19) UNE-EN 1990:2003. Eurocódigos. Bases de cálculo de estructuras. AENOR, 2003.

(20) UNE-EN 1991-2:2004. Eurocódigo 1: Acciones en estructuras. Parte 2: Cargas de tráfico en puentes. AENOR, 2004.

(21) International Standard ISO 2631-1:1997, Mechanical vibration and shock-Evaluation of human exposure to whole-body vibration - part 1: General requirements. 2nd Edition Genève: International Organization for Standardization, 1997.

(22) FIB. (2005). Bulletin 32: Guidelines for the design of footbridges. Fédération internationale du béton (FIB).

(23) Hivoss. (2008). Design of Footbridges. Guideline. Human Induced Vibrations of Steel Structure (Hivoss-RFS2-CT-2007-00033).

(24) Gómez M. (2004). A new and unusual cable-stayed footbridge at Valladolid (Spain), Steelbridge 2004: Symposium International sur les Ponts Metálliques, Milau, France, 23-25 June. 
(25) Ladret P., González A. (2005). Pretensado exterior en la pasarela del museo de la ciencia en Valladolid. Informes de la Construcción, 57(497): 5-12, doi:10.3989/ic.2005. v57.i497

(26) ARTeMIS. Structural Vibration Solutions: www.svibs.com.

(27) Cappellini, A., Manzoni, S., Vanali, M. (2012). Experimental and numerical studies of the people effects on a structure modal parameters. Proc. 30th International Modal Analysis Conference IMAC XXX, Jacksonville, USA.

(28) Ellis, B.R., Ji. T. (1997). Human-structure interaction in vertical vibrations, Proceedings of the ICE: Structures and Buildings, 122: 1-9.

(29) (2001). Dynamic performance requirements for permanent grandstands subject to crowd action. Interim guidance on assessment and design. The Institution of Structural Engineers.

(30) Porras J., De Sebastian J., Casado C., Lorenzana A. (2012). Modal mass estimation from output-only data using oscillator assembly. Mechanical Systems and Signal Processing, 26: 15-23.

(31) Brownjohn J. M. W., Pavic A. (2007). Experimental methods for estimating modal mass in footbridges using human-induced dynamic excitation. Engineering Structures, 29(11): 2833-2843.

(32) APS Dynamics. (2009). Instruction Manual: Electro-seis, model 400 shaker.

(33) López, M., Fernández, P., Brincker, R., Fernández, A. (2010). Scaling-factor estimation using an optimized mass-change strategy. Mechanical Systems and Signal Processing, 24(5): 1260-1273.

(34) Butz C. (2008). Codes of practice for lively footbridges: State-of-the-art and required measures. Proc. 3rd Int. Conf. Footbridge, Porto, Portugal. 